\title{
BER and PAPR Performance Analysis of MIMO-OFDM Systems Using Equalizers
}

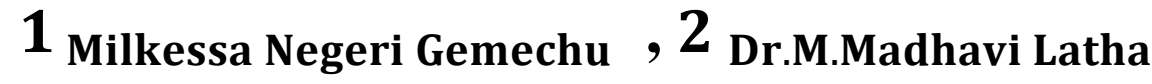 \\ $\mathbf{1}_{\text {milkynegeri@gmail.com, }} \mathbf{2}_{\text {mmadhavilatha@jntuh.ac.in }}$ \\ 1 PG Scholar: Dept. of ECE, Jawaharlal Nehru Technological University, Hyderabad \\ 2 Professor:Dept.of ECE, Jawaharlal Nehru Technological University, Hyderabad \\ DOI: 10.29322/IJSRP.11.01.2021.p10958 \\ http://dx.doi.org/10.29322/IJSRP.11.01.2021.p10958
}

\section{Abstract}

MIMO-OFDM (multiple input and multiple output Orthogonal Frequency Division Multiplexing) system is a new wireless broadband technology which became famous for being capable of high data rate transmission $\&$ robustness against multi-path fading. Fading effects must be mitigated at the receiver before demodulation, by using equalizer techniques. Equalizer is used to allow recovery of the transmit symbols, which is the major factor responsible for the BER. OFDM has many numbers of sub-carriers. Thus, the amplitude of the transmitted source signal has a large alternating or dynamic range. This results in a high Peak-to-Average Power Ratio (PAPR). In this paper, MMSE equalization and Zero Forcing equalization techniques are presented for reducing the bit error rate of the BPSK modulation technique. The BER performance of a clipping method for PAPR reduction technique is evaluated. In doing this, clipping technique helps for the reduction of PAPR for MIMO-OFDM. To reduce peak regrowth caused due to recursive/ iterative clipping, filtering technique is also used proceeding to the amplitude clipping operation.
Index Terms- BER, MIMO, OFDM, PAPR

\section{INTRODUCTION}

The principles of orthogonal frequency division multiplexing (OFDM) modulation have been in existence for about 7 decades time now. However, in a very recent years these multiplexing schemes have quickly moved out of textbooks and research laboratories into practical implementation. The techniques are implemented in data delivery systems over the Mobile phone or PSTN line, digital radio and television, and wireless networking systems.

Orthogonal frequency-division multiplexing (OFDM) is a technique of digital modulation in which a signal is divided into several narrow-band channels at various frequencies. Digital communications systems require each channel to operate at a specific frequency and with a Specific bandwidth.

OFDM is a sub-set of frequency division multiplexing; in which a single channel utilizes multiple sub-carriers on adjacent frequencies. In addition, the sub-carriers in an OFDM system are over-lapping to maximize the 
spectral efficiency. Ordinarily, overlapping adjacent channels can interfere with one another. However, subcarriers in an OFDM system are precisely orthogonal to each other.

Thus, they are able to overlap without interfering. As a result OFDM systems are able to maximize spectral efficiency without causing serious adjacent channel interference. OFDM is a unique case of FDM (Frequency Division Multiplexing). In FDM, the given bandwidth is subdivided among all the available subscribers or users. In this project, we will concentrate on the recent evolution of wireless communications systems, which is specifically OFDM and its detail operation mechanism including how to optimize its

\section{OFDM AND MIMO SYSTEM MODEL}

\section{a) OFDM System model}

OFDM is computationally highly efficient so that it utilizes modulation and demodulation techniques by using Fast Fourier Transform.

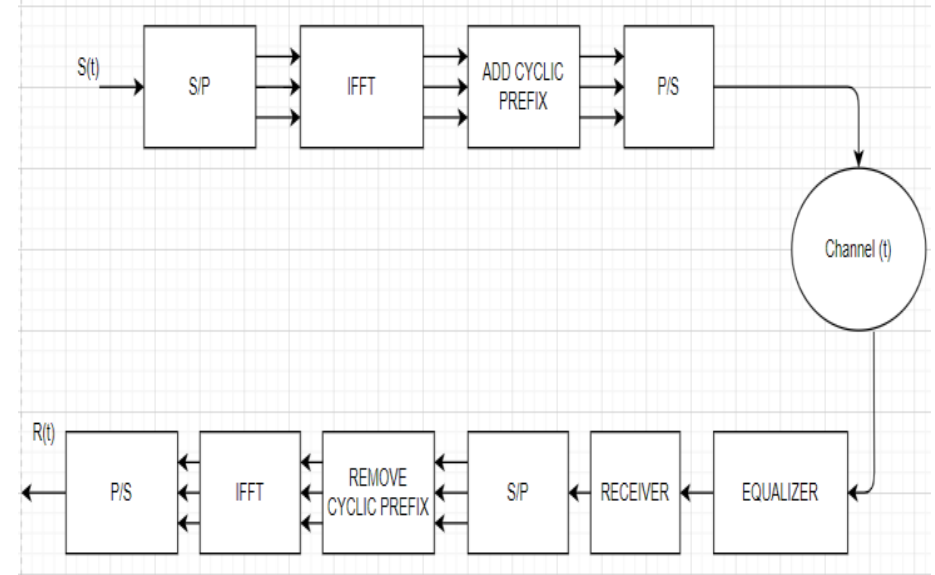

Figure 1 OFDM Block Diagram

.As can be seen from the above Figure 1 .
Serial to parallel converter, $\mathrm{S} / \mathrm{P}$, Transforms the data in each subcarrier form serial to parallel channel for simultaneous transmission in the channel.

Inverse Fast Fourier Transform, IFFT, Is used to produce orthogonal data subcarriers where the input data samples are modulated either by (e.g. BPSK) and after they are jointly correlated.

Cyclic Prefix, CP, Refers to the prefixing of a Symbol, with a repetition of the end.

The receiver discards this cyclic prefix, CP. It provides a guard interval to eliminate ISI from the previous symbol.Equalizer is used to remove the ISI imposed by the Channel.

Parallel to Serial converter P/S:_Converts the received serial channel signal to individual parallel subcarriers.

FFT: The FFT transform the cyclic prefix time domain signal to its equivalent frequency spectrum. A $2 X 2$ MIMO system is used for this project.

\section{Mathematical formula of OFDM signal.}

In OFDM systems, for frequency domain representations, let us consider data block of length $\mathrm{N}$ vector:-

$$
X=\left[X_{o} X_{1} X_{2} X_{3} X_{4} X_{5} \ldots \ldots X_{N-1}\right]^{T}
$$

And the duration of any symbol $X$ in the set.

$$
X_{o} X_{1} X_{2} X_{3} X_{4} X_{5} \ldots \ldots X_{N-1} \text { Is } T
$$

Which represents one of the subcarriers set.

* As the $\mathrm{N}$ subcarriers transmit the signal in orthogonal way as in the fig (1), we can have

$$
\boldsymbol{f}_{\boldsymbol{n}}=\mathbf{n} \Delta \boldsymbol{f}
$$

Where,

$$
\mathrm{n} \Delta f=\frac{1}{N T} \ldots \ldots \ldots \ldots \ldots \ldots \ldots \ldots \ldots
$$


* And NT is the duration of the OFDM data block $X$.

\section{b) A 2X2 MIMO OFDM Systems Model}

First of all, the input bit stream is fed into a convolution encoder. Then after the binary bits are assigned into various modulation techniques like ASK, BPSK and QPSK. The modulated digital signals are then fed into multiple input multiple output (MIMO) Encoder.

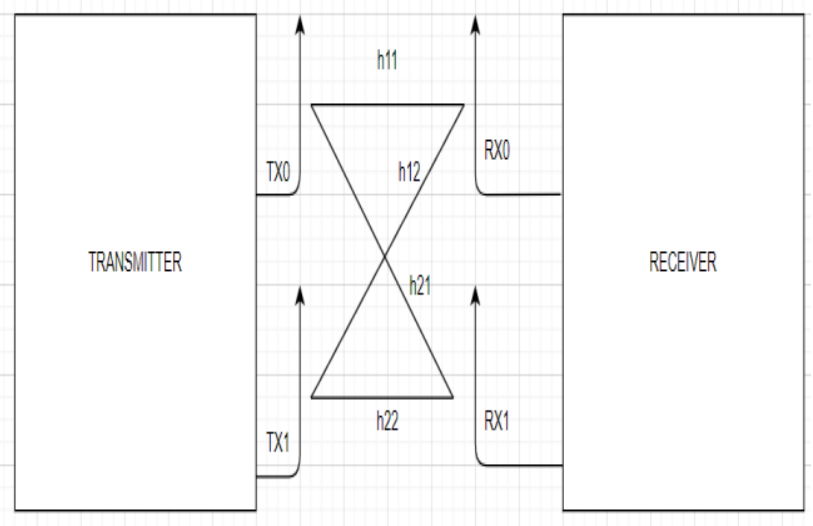

Figure 2. A 2X2 MIMO OFDM system block diagram

From the above diagram of $4 \mathrm{G} 2 \times 2$ MIMO, we can easily see that each receiver is getting two streams of data from the Transmitter 1 and the other, from transmitter 2 .

When we associate all the components in a single matrix; we will get Matrix channel or transmission matrix $H$ that could also be extended for $4 \times 4$ LTE MIMO. If we represent the Transmitters as $X$ and the Receiver components as Y, we will then we will obtain our equation for MIMO as:-

$$
\boldsymbol{Y}=\boldsymbol{H} \boldsymbol{x}
$$

Adding Gaussian noise and G, we will obtain our final equation:-

$$
\boldsymbol{Y}=\boldsymbol{H} \boldsymbol{x}+\boldsymbol{G}
$$

Writing Equation (5) in terms of transfer functions of each transmitter antenna:-

$$
\begin{aligned}
& y_{1}=h_{11} x_{1}+h_{12} x_{2}+n_{1} \\
& y_{2}=h_{21} x_{2}+h_{22} x_{2}+n_{2}
\end{aligned}
$$

\section{Single Carrier Modulation}

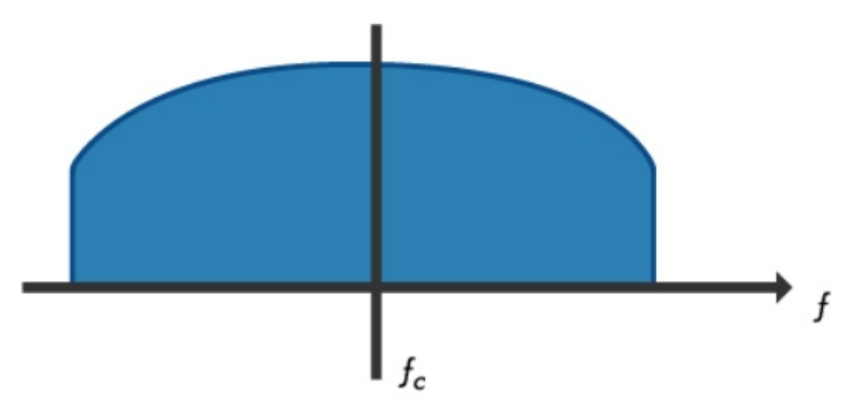

\section{Frequency Domain}

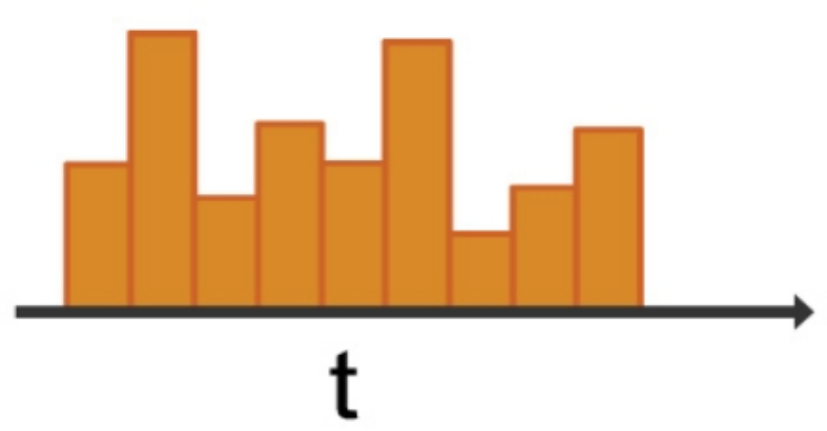

Time Domain

Figure 3. Single Carrier FDMA in both frequency and time domain 
Where $\boldsymbol{n}_{\mathbf{1}}$ and $\boldsymbol{n}_{\mathbf{2}}$ are noise of each transmission channels.

This technique of mixing signals allows greater resistance against interference like: - multipath, channel fading etc. Mathematical formula for the theoretically equivalent of channel capacity is referred from Shannon- Hartley theorem.

$$
\text { Capacity }=B W X \log _{2}(1+S N R)
$$

For the N number of transmitters in the MIMO system:

$$
\text { Capacity }=N X B W X \log _{2}(1+S N R)
$$

\section{c) Waveforms of Single carrier as compared to OFDM modulation}

OFDM technique belongs to the class of multi-carrier modulation schemes which uses several carriers in a single channel. OFDM decomposes the transmission band of frequency into a group of narrower contiguous sub-bands (carriers), and where each carrier is individually and distinctively modulated with dissimilar frequency.

You can implement this type of modulation with an inverse Fast Fourier transform (IFFT) by using narrow orthogonal sub-carriers, the OFDM signal gains a robustness over a frequency-selective fading channel and eliminates adjacent frequency's sub-carrier crosstalk.

At the receiving end, we de-modulate the OFDM signal with a Fast Fourier transform (FFT) and equalize it with a complex gain at each sub-carrier.

As multiple data streams can be transmitted simultaneously with multiple carriers, OFDM is not influenced by the noise in the same extent as singlecarrier modulation (See Figure 4). This is due to the fact that time per symbol can be lengthened by the number of carriers.

\section{Multiple Carrier Modulation}

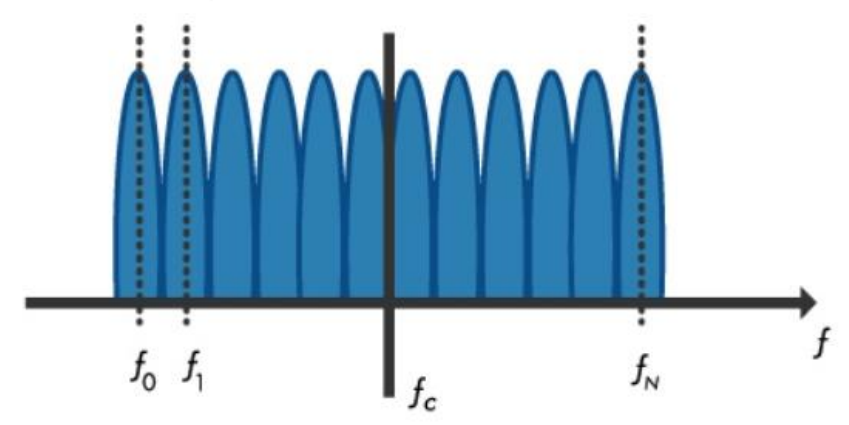

Frequency Domain
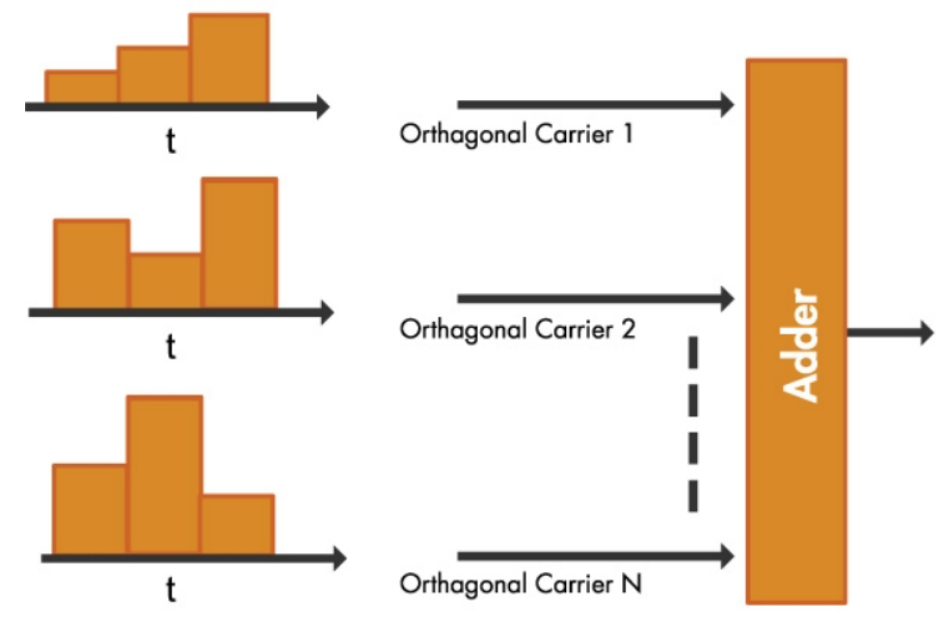

Time Domain

Figure 4. Multiple carrier OFDM Signal in Time and Frequency domain.

As observed on Figure 5 below, In OFDM, when the amplitude of individual sub-carrier reaches the maximum, the carriers are arranged at intervals of 1 / symbol time in such a way that the amplitude of other subcarriers is 0 , therefore preventing interference between symbols. 


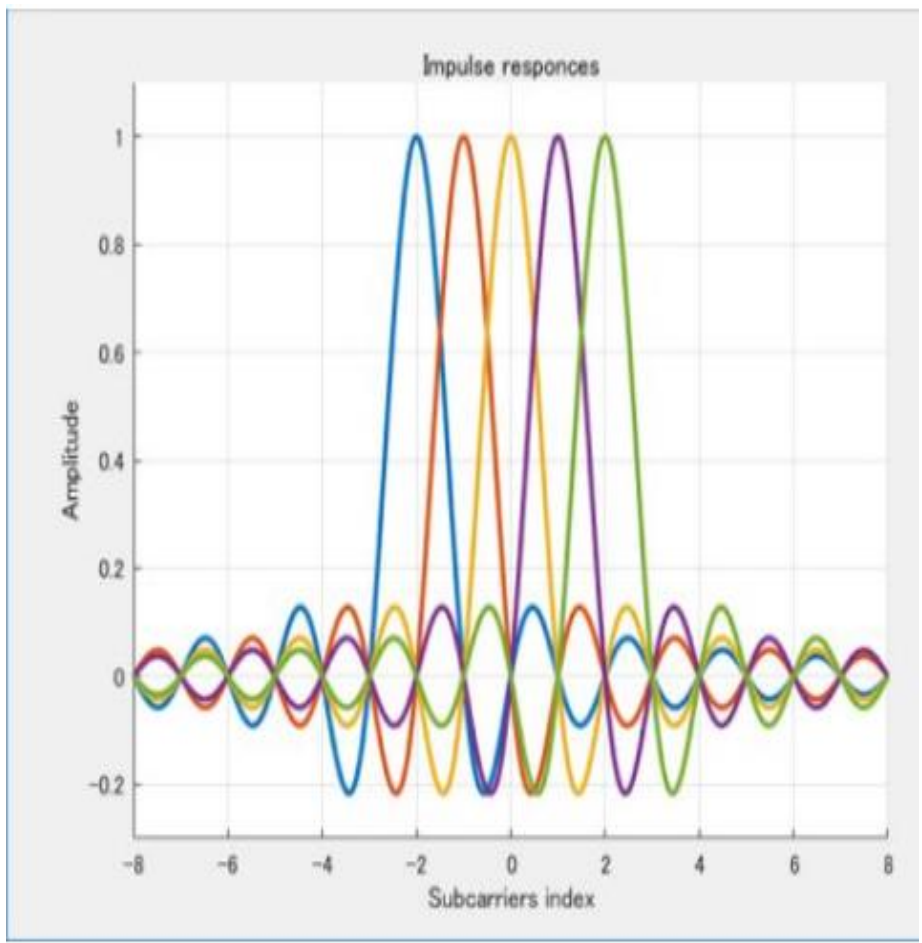

Figure 5. Frequency domain representation of orthogonal subcarriers in an OFDM waveform.

The time difference of arrival between the direct wave and the reflected wave increases significantly when the signal is transmitted over a long range of time (See Figure 6 below). In that situation, the number of subcarriers is larger than in a smaller service range.

Multi-carrier transmission in an OFDM systems is very effective in multi-path environments. This is due to the fact that the influence of multi-path is concentrated only on unique subcarriers as compared to a single-carrier transmission.

\begin{tabular}{|c|c|c|c|}
\hline Es Sectinat Aneyeer & & 0 & $x$ \\
\hline the Jool Xen Blopad Helo & & & , \\
\hline
\end{tabular}

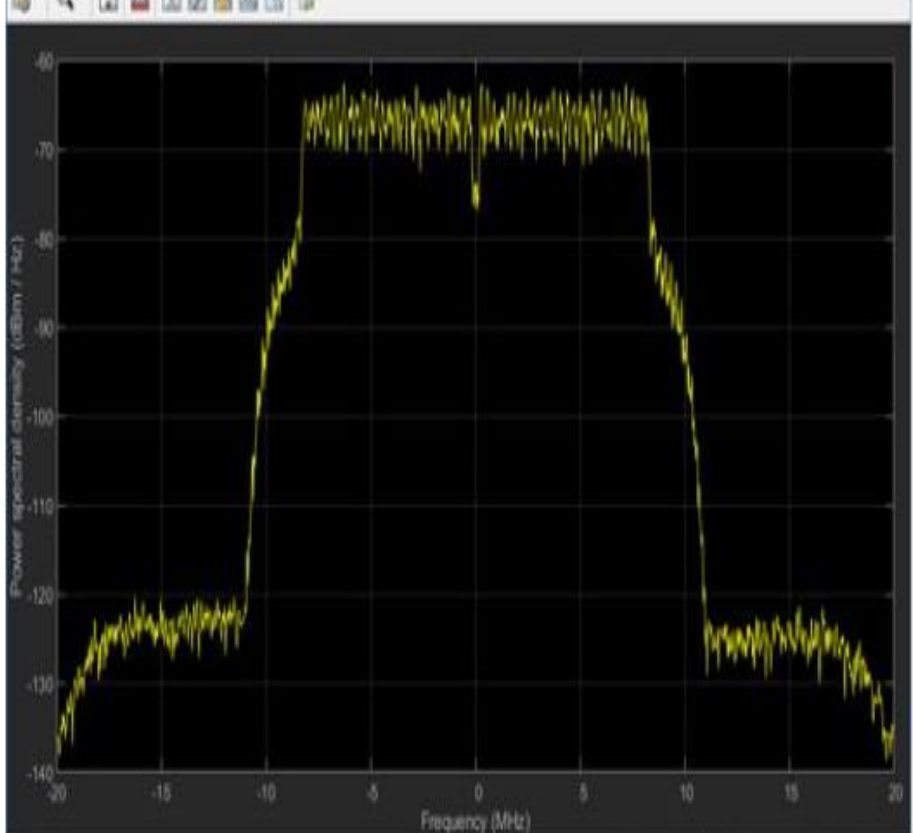

pasowey

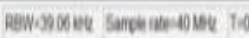

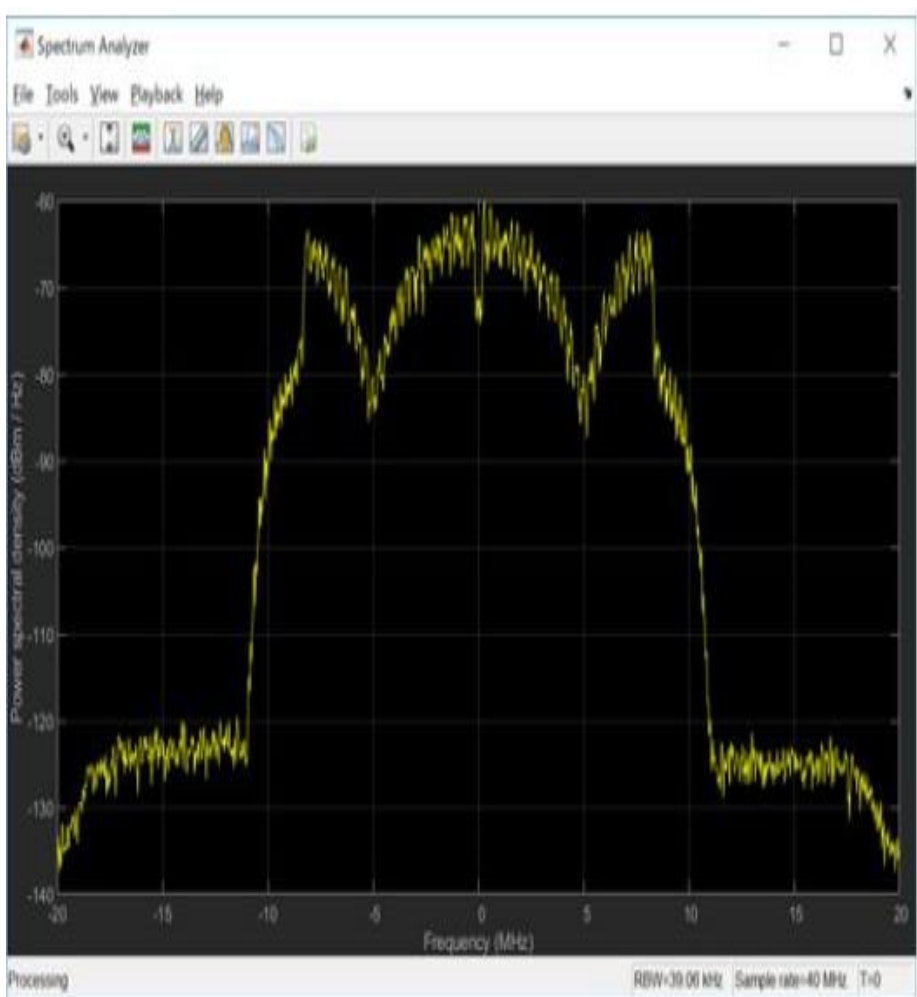

Figure 6. OFDM waveform influenced by multipath and Ideal OFDM waveform 
III. Bit Error Rate, BER, and Peak to Average Power Ratio, PAPR

a) Bit Error Rate (BER): Is defined as the ratio of number of bits which are having errors divided by the total number of transferred bits during a certain interval of time. Bit Error Rate is a unit-less performance measure, mostly expressed in percentage.

BER $=\frac{\text { Total number of Bits received in Error }}{\text { Total number of bits transmitted }} \ldots$

The analytical BER expressions for M-ary QAM signalling in AWGN and Rayleigh Channels are respectively given by the following equations:-

$$
\begin{gathered}
P_{e}=\frac{2(M-1)}{M \log 2^{M}} \mathbf{Q}\left(\sqrt{\frac{6 E_{b}}{N_{0}}} \cdot \frac{\log 2^{M}}{M^{2}-1}\right) \ldots \ldots \\
P_{e}=\frac{(M-1)}{M \log 2^{M}}\left(1-\sqrt{\frac{3 \gamma \log _{2} \frac{M}{M^{2}-1}}{3 \gamma \log _{2} \frac{M}{\left(M^{2}-1\right)+1}}}\right) \ldots \ldots \ldots
\end{gathered}
$$

Where $\gamma$ and $\mathrm{M}$ denote $\frac{\boldsymbol{E}_{\boldsymbol{b}}}{\boldsymbol{N}_{\boldsymbol{o}}}$ and the modulation order respectively. While $\mathrm{Q}(\mathrm{x})$ is defined as the standard $\mathrm{Q}$ function defined as :

$Q(x)=\frac{1}{2 \pi} \int_{x}^{\infty} e^{\frac{-t^{2}}{2}} d t \ldots \ldots \ldots \ldots \ldots \ldots \ldots \ldots \ldots \ldots \ldots \ldots \ldots \ldots$

\section{b) Peak to Average Power Ratio (PAPR) reduction}

PAPR can be described as the relationship among the maximum strength of a pattern in a transmit OFDM image and its average strength. It is caused due to the huge variety of sub carriers in OFDM systems.
$P A P R_{d b}=10 \log _{10} \frac{P_{\text {peak }}}{P_{\text {average }}}=\frac{\max |x(t)| x^{*}(t)}{E\left|x(t) x^{*}(t)\right|}$

.......(16)

Where $\boldsymbol{P}_{\text {peak }}$ and $\boldsymbol{P}_{\text {average }}$ are the peak and average power of a given OFDM symbol. E denotes the expected value; $X_{n}$ denotes the transmitted OFDM signals. If the number of sub carrier's t increased, the PAPR power also increases.

\section{c) Clipping}

Clipping is the easiest technique to reduce the power by setting a maximum level for the transmitted signal. We can use filtering operation to decrease the appearance of the out-of-band radiation. The signal $x^{p}[\mathrm{~m}]$ is the pass-band modulated one with carrier frequency $f_{c}$. We symbolize the clipped form of the pass-band modulated signal as $x_{c}^{p}[m]$. The expression of this signal is:

$x_{c}{ }^{p}[m]=\left\{\begin{array}{lc}-A ; & x^{p}[\mathrm{~m}] \leq-A \\ x^{p}[\mathrm{~m}] ; & x^{p}[\mathrm{~m}]<A \ldots(17) \\ A & x^{p}[\mathrm{~m}] \geq=A\end{array}\right.$

Where the clipping level is denoted by A and (CR) is the clipping ratio that can be represented as follows:

$$
\boldsymbol{C R}=\frac{\boldsymbol{A}}{\boldsymbol{\sigma}}
$$

In this project, clipping ratio is taken as 0.79 . While the symbol $\sigma$ Is RMS value of OFDM signal such that:

$$
\sigma=\sqrt{N}
$$

For the baseband and

$$
\sigma=\sqrt{N / 2}
$$

For the pass band OFDM signal. $\mathrm{N}$ is the number of subcarriers in the OFDM signal. 


\section{d) Filtering}

In this project, PAPR were reduced by clipping the peak amplitude of the transmitted signal and making it to pass through a low pass filter ( See Figure 3.) thereby getting a response in the time domain.

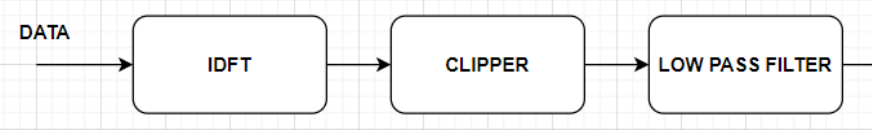

Figure 7. OFDM System with Nyquist rate

\section{Clipping and time domain filtering}

For the case of over sampled signal; band pass filter (BP) is required to minimize the out of band radiation. However, for the band limited signal clipped at Nyquist sampling rate; all the distortions fall within the band. Thus, a low pass filter is sufficient.

PAPR could also be reduced by using clipping and frequency domain filtering as shown

(Figure 4) below. To reduce peak regrowth caused by filtering recursive/ iterative clipping and filtering techniques were also used.

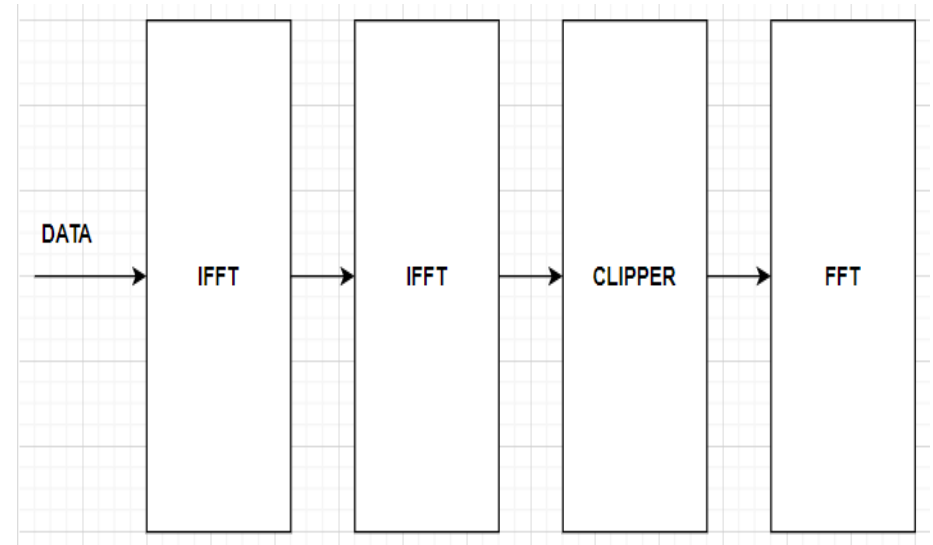

\section{Figure 8. Clipping and Filtering in}

The Clipped version of the signal as seen on Figure 4 is expressed using the following equation:-

$$
x_{c}^{p}[m]=\left\{\begin{array}{c}
-A, x_{p}[m] \leq A \\
x_{p}[m], \quad x_{p}[m]<A \ldots \ldots \ldots \\
A, x_{p}[m] \geq A
\end{array}\right.
$$

PAPR is also obtained as a clipping ratio (CR); which could be defined as the clipping level, normalized by the Root mean square, RMS value, $\sigma$, of OFDM signal given as in equation:

$$
C R=\frac{A}{\sigma}
$$

\section{Zero Forcing (ZF) and Minimum Mean Square Error (MMSE) Equalizers}

The equalizer is a linear filter that gives an approximate inverse of the channel transfer function response. Provided that it is common for the channel characteristics to be unknown or to change over time, the usual behavior of an equalizer is a structure that is adaptive in nature.

The multi-path channel results in frequency selectivity and Inter-symbol Interference. Equalization can reduce the Inter-symbol Interference and noise effects for improved demodulation.

Inter- Symbol Interference

This is a type of signal distortion, in which one or more symbols interfere with consecutive signals, resulting in noise or delivering a poor quality output at the receiver. The main causes of Inter-symbol Interference are:-

$>$ Multi-path Propagation

Non-linear frequency in channels.

\section{a) Zero Forcing equalizer}


The Zero - Forcing Equalizer implements the inversed form of the transmission channel transfer function to the received signal, and therefore restore the signal that was before the channel; at the receiver end. Not useful for most practical applications.

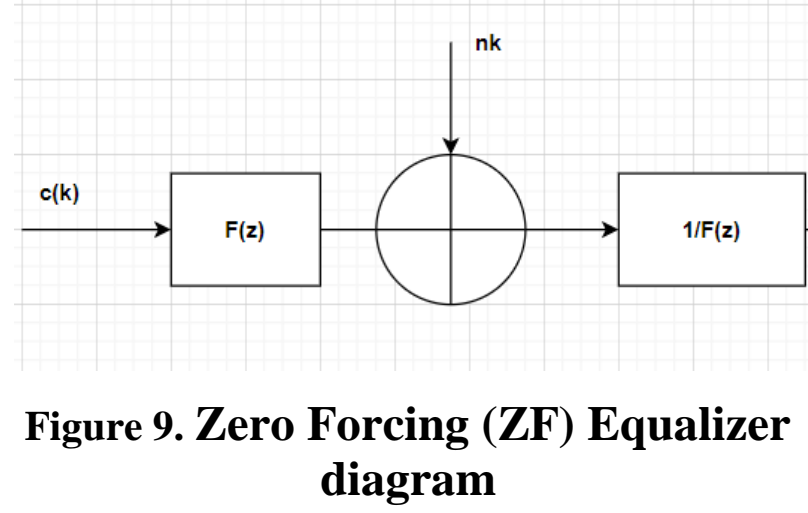

For a channel with frequency response $F(Z)$, the zero forcing equalizer $\mathrm{C}(\mathrm{f})$ is constructed so that: - As seen in the above figure, Figure (5)

$$
C(f)=\frac{1}{F(Z)}
$$

Where $c(k)$ is input, $c^{\prime}(k)$ is output and $n k$ is the channel noise. Thus, Combination of channels and equalizer gives a flat frequency response and linear phase.

$F(Z) C(f)=1$

\section{b) Minimum Mean Squared Error (MMSE)}

To reduce the inter-symbol interference, ISI, and additive noise consequences, the equalizer coefficients can be optimized by using the minimum mean squared errors (MMSE) technique.

MMSE Does not usually eliminate ISI completely but reduces the power of Noise \& ISI components.
When the Signal to Noise Ratio has elevated values, the MMSE equalizer as shown in the following (Figure 6 ) works as Zero Forcing does, but whilst the SNR has lower values, the fact that MMSE equalizer takes under consideration the noise and sign variance, makes to now not extend the noise as Zero Forcing does.

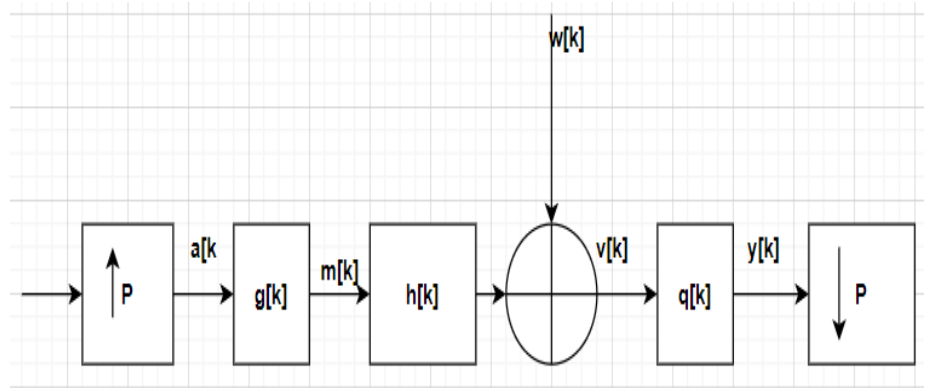

As seen on Figure 2.2. Let us extract the two symbols which interfered with each other. In the first time slot, the received signal on the first receive antenna is, the outputs of the $2 \times 2$ MIMO OFDM is mathematically expressed as:-

For the First Receive antenna

$$
y_{1}=h_{11} x_{1}+h_{12} x_{2}+n_{1}
$$

For the second receive antenna:

$$
y_{2}=h_{21} x_{1}+h_{22} x_{2}+n_{2}
$$

For simplicity, the above equations can be rewritten in matrix format as follows:-

$$
\left[\begin{array}{l}
y_{1} \\
y_{2}
\end{array}\right]=\left[\begin{array}{ll}
h_{1,1} & h_{1,2} \\
h_{2,2} & h_{2,1}
\end{array}\right]\left[\begin{array}{l}
x_{1} \\
x_{2}
\end{array}\right]+\left[\begin{array}{l}
n_{1} \\
n_{2}
\end{array}\right]
$$

Equivalently,

$$
y=H x+n
$$

The Minimum Mean Square Error (MMSE) technique tries to search for a coefficient $\mathrm{W}$ which minimizes or reduces the criterion.

$$
E\left\{\left[W_{y}-x\right]\left[W_{y}-x\right]^{H}\right\}
$$


After Solving the above equation, we get:-

$W_{m m s e}=\left[H^{h} H+N_{0} I\right]^{-1} H^{H}$

When the noise term is entirely zero, the Minimum mean square equalizer, MMSE, equalizer of eq. (29) minimizes to the level of Zero Forcing equalizer.

The equalizer, $\boldsymbol{W}_{\boldsymbol{m m s} \boldsymbol{e}}$ gives an estimate of the transmit signal vector in a way that the mean square error between the two is minimum.

As seen on the Figure 6 above, for a trivial channel (i.e., $\tilde{h}[\mathbf{k}]=\delta[\mathbf{k}]$ ) and white noise $\{\tilde{w}[\mathbf{k}]\}$,

$\checkmark$ Here we design the minimum mean-squared error (MMSE) equalizer coefficients $\{\mathbf{q}[\mathbf{k}]\}$;

$\checkmark$ assuming that the input symbols $\{\mathbf{a}[\mathbf{n}]\}$ and the noise $\{\tilde{w}[\mathbf{k}]\}$ are white random sequences that are uncorrelated with each other:

$$
\begin{array}{r}
\left\{\boldsymbol{a}[\boldsymbol{m}] \boldsymbol{a}^{*}[\boldsymbol{n}]\right\}=\boldsymbol{\delta}_{\boldsymbol{a}}^{2} \boldsymbol{\delta}[\boldsymbol{m}-\boldsymbol{n}] \\
E\left\{\widetilde{w}[\boldsymbol{k}] \widetilde{w}^{*}[\boldsymbol{l}]\right\}=\boldsymbol{\delta}_{\boldsymbol{w}}^{2} \boldsymbol{\delta}[\boldsymbol{k} \ldots \ldots \\
E\left\{\boldsymbol{a}[\boldsymbol{m}] \overline{\boldsymbol{w}}^{*}[\boldsymbol{l}]\right\}=\ldots \ldots \ldots \ldots \ldots \ldots
\end{array}
$$

Thus, our goal is to make:

$$
\begin{aligned}
& \mathbf{y}[\mathbf{m}] \approx \mathbf{a}[\mathbf{m}-\Delta], \text { for some integer } \Delta \geq 0 \ldots . . \text { (34) }
\end{aligned}
$$

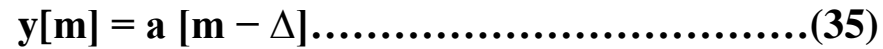

We are now able to make perfect decisions on sequences $\mathrm{a}[\mathrm{m}]$.We would never expect a perfect output in the presence of noise Thus, we take as our objective the minimization of the error signal.

$\mathbf{e}[\mathbf{m}]:=\mathbf{y}[\mathbf{m}]-\mathbf{a}[\mathbf{m}-\Delta]$.

In particular, we minimize the mean squared error

(MSE):

$$
\varepsilon:=E\left\{|e[m]|^{2}\right\}
$$

\section{SIMULATION RESULTS}

With the wide availability of advanced computational platforms, different analysis and various simulation tools, computer oriented simulations have become a highly available means to demonstrate and analyze the operations of wireless communication systems by the consecutive stages of development such as conceptualization, designing, building hardware, verifying and validating. In this project a simple simulations of the MIMO OFDM systems under consideration is presented. This will primarily serve to check the feasibility of implementation of equalizers to a MIMO system that may be deployed for OFDM. MATLAB, an advanced technical computing language has been used for simulating and analyzing of our model. Figure 5.1 presents a simple and easily understandable level block diagram of the simulation model.

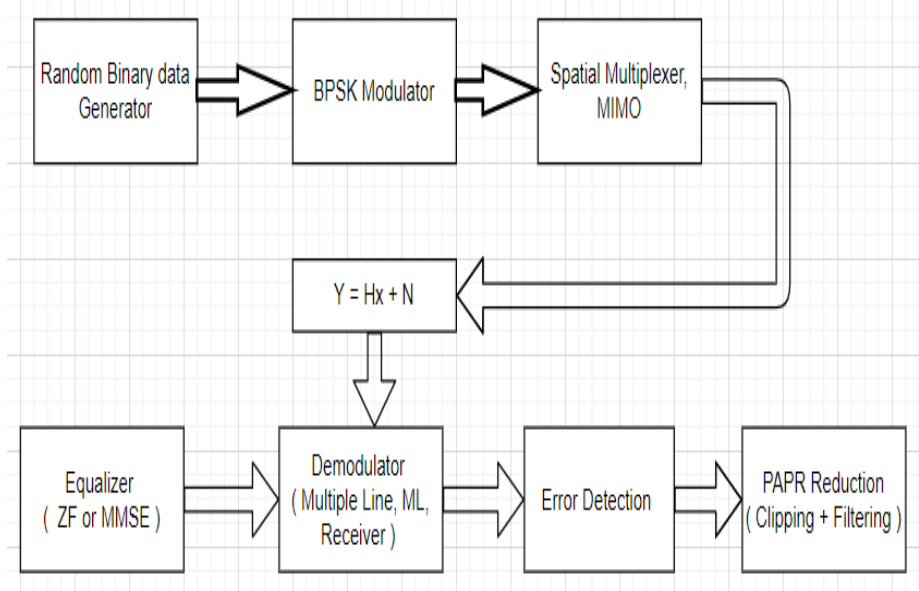

Figure 11. Block Diagram of the Simulated

\section{Model}

This project is a simulation study on the performance comparison of 2X2 MIMO-OFDM with different Equalizers, using BPSK modulation technique. The BER performance is investigated in this comparison using MATLAB code. The definition of the BER can be explained as the effected bits divided by the whole number of the transmitted bits over a time interval. This calculation provides us with a percentage quantity that 
is a unit-less. The code has been developed successfully for simulation and eight SNR points has been targeted by two equalizers on 2x2 MIMO System. The performance of $\mathrm{ZF}$ and MMSE is compared from simulation graph and MMSE is found to be having better performance. Also PAPR of Single Carrier, SCFDMA, and OFDMA is calculated by applying Clipping method. Filtering helps to remove out of band radiation that is left after Clipping.

a) Comparison of Graph of BER and PAPR of MIMO OFDM Systems Using ZF and MMSE Equalizers

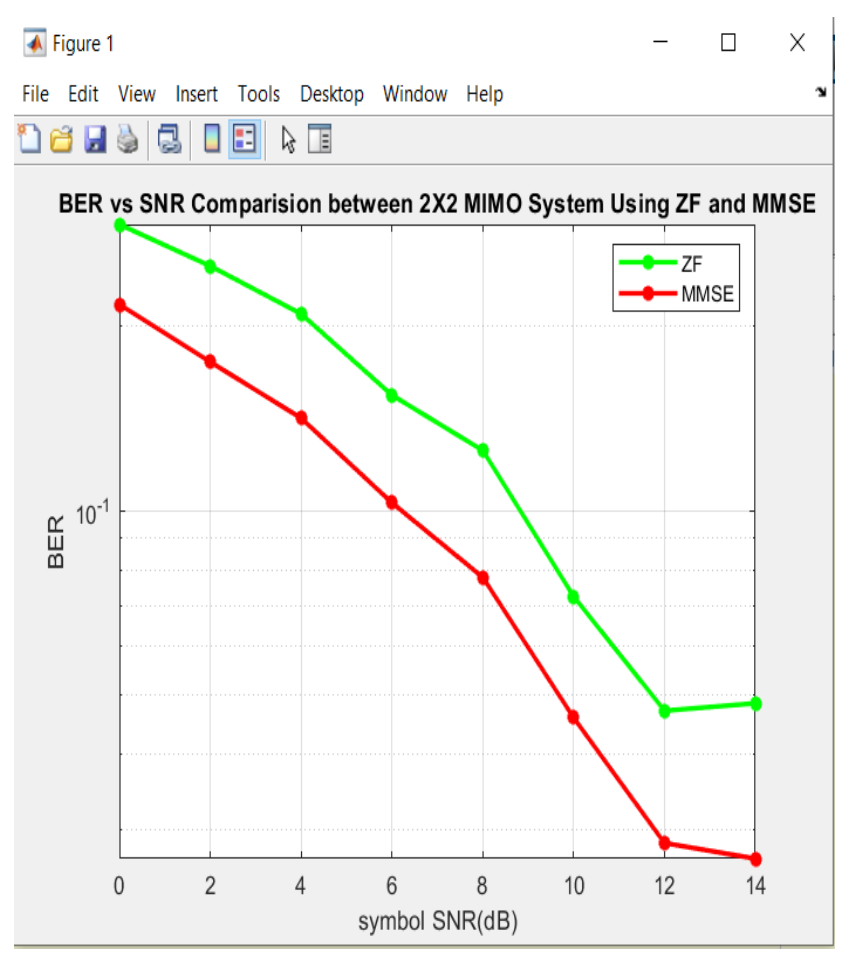

Figure 12. BER vs SNR Comparison of $2 \times 2$ MIMO OFDM system using ZF and MMSE Equalizers

As depicted on Figure 12, above, let's see the tabulated BER vs SNR of ZF and MMSE equalizers at each of the marked points on the simulation graph; to see which one has better performance.

Table 5.1 Comparison of BER vs SNR of MIMO OFDM with ZF and MMSE Equalizers

\begin{tabular}{|c|c|c|c|}
\hline Equalizer & SNR & BER & Eb/No dB \\
\hline ZMSE & & 0.2854 & 0 \\
\hline ZF & 2 & .2539 & .5078 \\
\hline MMSE & & .1765 & .353 \\
\hline ZF & 4 & $\mathbf{0 . 2 0 0 5}$ & .802 \\
\hline MMSE & & $\mathbf{0 . 1 3 9 8}$ & .5592 \\
\hline ZF & 6 & $\mathbf{0 . 1 0 3}$ & .618 \\
\hline MMSE & & $\mathbf{0 . 1 5 5 9}$ & .9354 \\
\hline ZF & $\mathbf{8}$ & $\mathbf{0 . 0 7 8 2}$ & .6256 \\
\hline MMSE & & $\mathbf{0 . 1 2 2 4}$ & .9792 \\
\hline
\end{tabular}

As can be seen from the Table 5.1. Above, The BER and $\mathrm{Eb} / \mathrm{No} \mathrm{dB}$ of $\mathrm{ZF}$ equalizer, at each SNR point, is generally greater than that of MMSE equalizer at each point of SNR.

Additionally, from the same table above, the following observations are made:-

The MMSE equalizer's performance is the better than $\mathrm{ZF}$ equalizer, as it provide relatively minimum BER value for corresponding SNR.

On the other hand, the lower SNR for the same BER implies that it consumes much less power than the other technique.

Hence we can conclude that BER performance of 
MMSE Equalizer is superior to ZF Equalizer.

\section{b) PAPR of SC-FDMA and OFDMA using BPSK modulation.}

\section{PAPR Calculation for SC-FDMA \& OFDM}

PAPR occurs in a multi-carrier system where the different sub-carriers are out of phase with each other. At each instant they are different with respect to each other, at different phase values.

When all the points achieve the maximum value simultaneously; this will cause the output envelope to suddenly shoot up, which causes a 'peak' in the output envelope. A high PAPR significantly degrades the transmit signal quality by changing the constellation nature of the transmit signal \& thus leads to in-efficient operation.

From Simulation graph of Figure 13. PAPR:

Upon application of clipping method, the graph of Single Carrier Frequency Division Multiple Access, SC-FDMA, is swiftly descending and;

Therefore have lower PAPR than that of OFDM when evaluated at nearly same points as per Table 2 .

Thus, PAPR of OFDM is greater than that of SCFDMA. This is due to the presence of large number of independently modulated sub-carriers in an OFDM system, the peak value of the system can be very high, as compared to the average of the whole system.

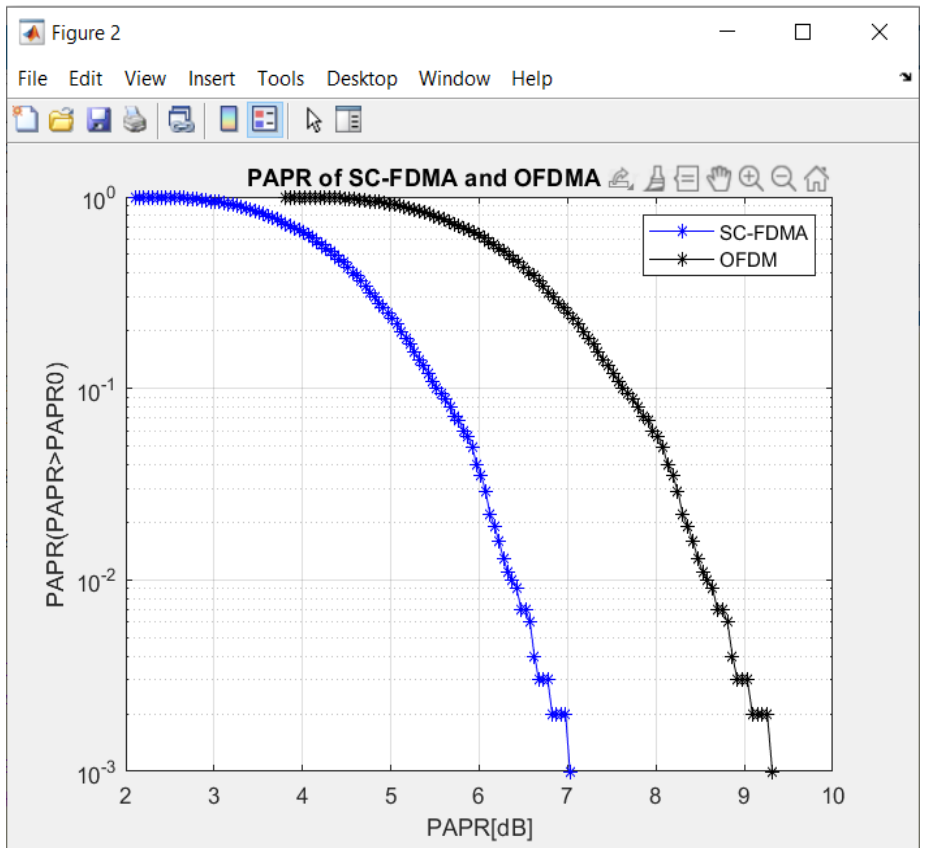

Figure 13. PAPR reduction using clipping method for SC- FDMA and OFDMA in BPSK modulation

On the above graph of Figure 13. The vertical axis represents PAPR which is out of band portion of the Amplitude; as it decrease from zero at the top left, to $10^{-3}$ at the bottom left corner and It's labelled as X axis on the corresponding table

In the table below, comparison of both multiplexing schemes are tabulated as evaluated from Figure 9.

See the following table 2. For the values at each point. 
Table 5.2. PAPR (Peak to Average power ratio) of SC-FDMA and OFDM

\begin{tabular}{|c|c|c|c|}
\hline & $X(d B)$ & $\mathbf{Y}$ & Product (dB) \\
\hline SC-FDMA & 1.687 & 0.998 & 1.684 \\
\hline OFDM & 1.687 & 1 & 1.687 \\
\hline SC-FDMA & 4.011 & 0.653 & 2.619 \\
\hline OFDM & 4.004 & 0.995 & 3.984 \\
\hline SC-FDMA & 5.542 & 0.102 & .565 \\
\hline OFDM & 7.614 & 0.109 & .8299 \\
\hline SC-FDMA & 6.45 & 0.01 & .0645 \\
\hline OFDM & 9.266 & 0.001 & .009266 \\
\hline SC-FDMA & 7.707 & 0.001 & .007707 \\
\hline
\end{tabular}

From Simulation graph of PAPR as shown on Figure 9 and Table 5.2, we get the following observations Upon application of clipping method the graph of SCFDMA is swiftly descending and therefore have lower PAPR than that of OFDM when evaluated at nearly same points as per table 2 .

* Thus, PAPR of OFDM is greater than that of SC- FDMA. This is due to the presence of large number of independently modulated sub- carriers in an OFDM system, the peak value of the system can be very high as compared to the average of the whole system.

c) BER and PAPR performance analysis of MIMO OFDM systems; using Rayleigh Channel, Without

\section{Equalizers.}

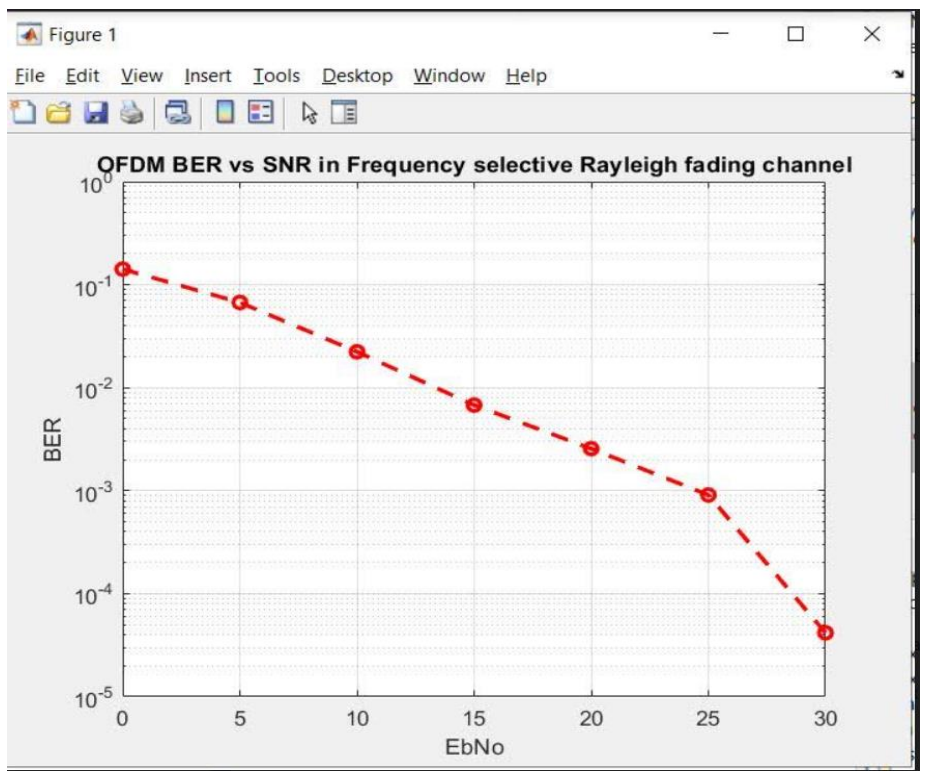

Figure 14. BER vs SNR performance analysis of MIMO OFDM, using Rayleigh Channel, without equalizer

As we easily observe from the above graph of Figure 10, the BER performance of MIMO OFDM without equalizer is higher than that of with equalizer; when evaluated at the same SNR values of $5,10, \ldots$ for both Figures (; See Figure 9 for comparison). Therefore this is not preferable as we want minimum error (i.e. less BER) which means minimum Inter-symbol Interference. 
d) Comparison of BER vs SNR, in MIMO OFDM system, using Rayleigh and AWGN theoretical channel, with and without equalizer, respectively.

Here, AWGN is with equalizer, while Rayleigh theoretical and simulated are without equalizers.

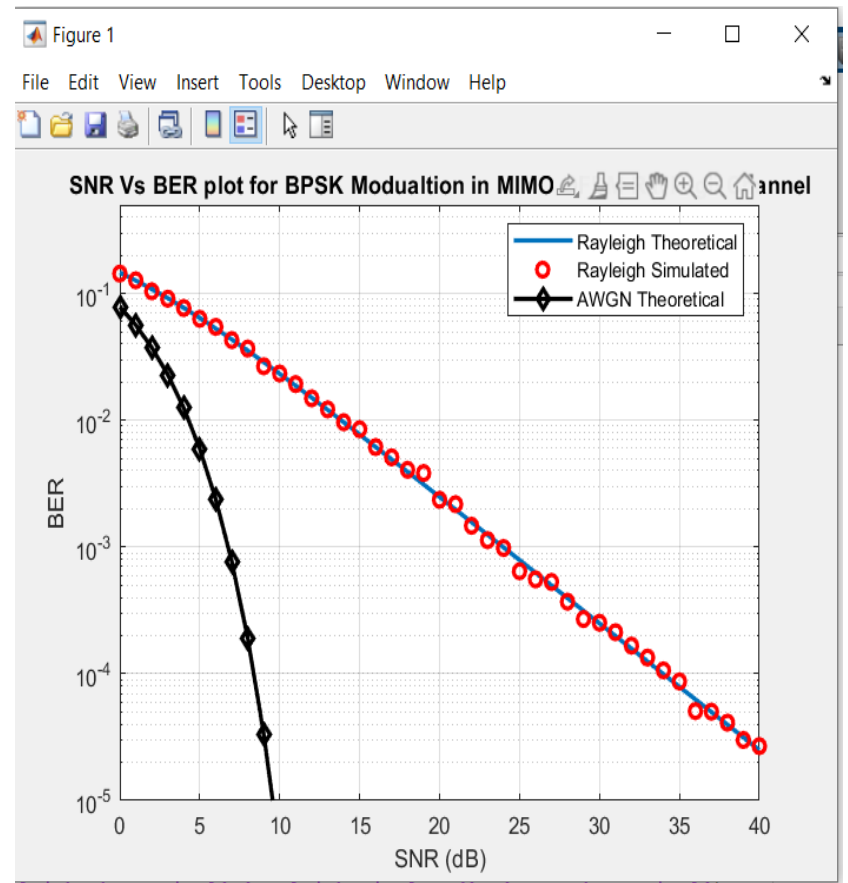

Figure 15. Comparison of BER vs SNR, in MIMO OFDM system, using Rayleigh and AWGN theoretical channel, with and without equalizer, respectively.

As can be seen from the graph, under the given parameters, the BER vs SNR of AWGN theoretical, channel is minimum than that of Rayleigh simulated and Rayleigh Theoretical. Therefore, it's more preferable.
Comparing Rayleigh Simulated and Rayleigh Theoretical, the BER vs SNR of Rayleigh simulated is less than that of Rayleigh simulated. Thus, Rayleigh Simulated is more preferable than Rayleigh theoretical.

\section{CONCLUSION}

In this paper, BER vs SNR of MIMO OFDM systems has been calculated and simulated with and without equalizer. As can be seen from the plotted graphs, the BER vs SNR performance of MIMO OFDM systems with equalizer is minimum as compared to the plotting with ZF and MMSE equalizer. PAPR of MIMO OFDM systems using amplitude Clipping and then followed by filtering has also been calculated and plotted. Filtering has enabled us reduce peak regrowth caused by iterative clipping.

The performance of BER vs SNR of MIMO OFDM under two different channels; AWGN and Rayleigh channel are compared and their graphs analyzed. When the performance of two different equalizers are evaluated for BER vs SNR and PAPR; Minimum Mean Square Error, MSE, has been found to operate far better than Zero Forcing Equalizer, ZF. And under the given specific parameters; AWGN channel is found to have better performance when it comes to getting minimum BER vs SNR.

The PAPR performance of Single channel frequency division multiplexing, SC_FDM, and orthogonal frequency division multiplexing, OFDM has been compared from the plotted diagram. SC- FDM is found to be having less PAPR than OFDM at each point along 
the two dimensional axis of PAPR0 and PAPR (x, y). PAPR0 is the normal signal power under no out of band distortion. SC-FDM performed better due to lack of multiple carriers in this case, as it is only along a single line.

\section{RECOMMENDATION}

During the task of entire paper work, we have seen BER and PAPR performance analysis of MIMO OFDM systems only under AWGN and Rayleigh channel. And also we have used only Zero Forcing and Minimum Mean Square Error. Therefore, we would like to suggest the readers to try to work under different set of conditions i.e. Using Wireless channel models different from AWGN and Rayleigh Channel. And also we would like to suggest to use different Equalizers than Zero Forcing and Minimum Means Square Error. It's also suggested for the readers to use different PAPR reduction techniques, like Block coding, Selective Level Mapping (SLM), Partial Transmit Sequences (PTS) and others. Then compare the result with what we have got in this work by using Clipping and Filtering than Clipping. Finally, it is advised to compare and analyze the results that are acquired under different conditions with respect to the results that are presented in this paper work.

\section{REFERENCES}

[1] Kai-Kit Wong; Ross D. Murch; Khaled Ben Letaief, "Performance Enhancement of Multiuser MIMO Wireless Communication Systems", IEEE Transactions on Communications, vol. 50, NO. 12, December 2002.

[2] Blum, Rick S.; Ye Li; Jack H. Winters; Qing Yan, "Improved space-time coding for MIMO-OFDM wireless communications", IEEE Transactions on Communications, vol. 49, no. 11, pp. 1873-1878, 2001. [3] Foschini, Gerard J.; Michael J. Gans, “ On limits of wireless communications in a fading environment when using multiple antennas", Wireless personal communications, Springer, vol. 6, no.3, pp. 311-335.

[4] Chan, Albert M.; Gregory W. Wornell, “A class of block-iterative equalizers for inter-symbol interference channels", In Communications, ICC 2000, IEEE International Conference on, vol. 1, pp. 31-35, 2000.

[5] Chalise, Batu K.; Luc Vandendorpe, "Performance analysis of linear receivers in a MIMO relaying system", Communications Letters, IEEE, vol. 13, no. 5, pp. 330-332, 2009.

[6] A. John, Multicarrier Modulation for Data Transmission: An Idea Whose Time Has Come. IEEE Communications Magazine, 1990.

[7] S. Albdran, A. Alshammari, and M. Matin, The Sensitivity of Bit Error Rate (BER) Performance in Multi-Carrier (OFDM) and Single-Carrier, SPIE optics and photonics, 2012.

[8] T. Hwang, C. Yang, G. Wu, S. Li, G. Y. Li, “OFDM and its wireless applications: A survey", IEEE Transactions on Vehicular Technology, Volume: 58, Issue: 4, Page(s): 1673 - 1694, May 2009 
[9] Y. S. Cho, W. Y. Yang, MIMO-OFDM Wireless Communications with MATLAB, John Wiley \& Sons Publication, 2010

[10] M.V.R. Vittal, "Performance enhancement of OFDM signals using PAPR reduction techniques and the comparison of their performance", International Journal of Computer Applications, IJCA Journal, Volume 41 - Number 19, 2012

[11] H. Yang, "A road to future broadband wireless access: MIMO-OFDM-Based air interface", IEEE Communications Magazine, Volume: 43, Issue: 1, Page(s): 53 - 60, Jan. 2005

[12] M. Jiang and L. Hanzo, "Multiuser MIMO-OFDM for next generation wireless systems", Proceedings of IEEE, Volume: 95, Issue: 7, page(s): 1430-1469, July 2007

[13] Y. Wu, JWM Bergmans, S. Attallah, "Carrier frequency offset estimation for multiuser MIMOOFDM uplink using CAZAK sequences: Performance and sequence optimization", EURASIP Journal on Wireless Communication and Networking, 2011

[14] Allert van Zelst and Tim C. W. Schenk, "Implementation of a MIMO OFDM based Wireless LAN system," IEEE Trans. Signal Processing, vol. 52, No. 2, pp. 483-494, Feb. 2004.

AUTHORS: 


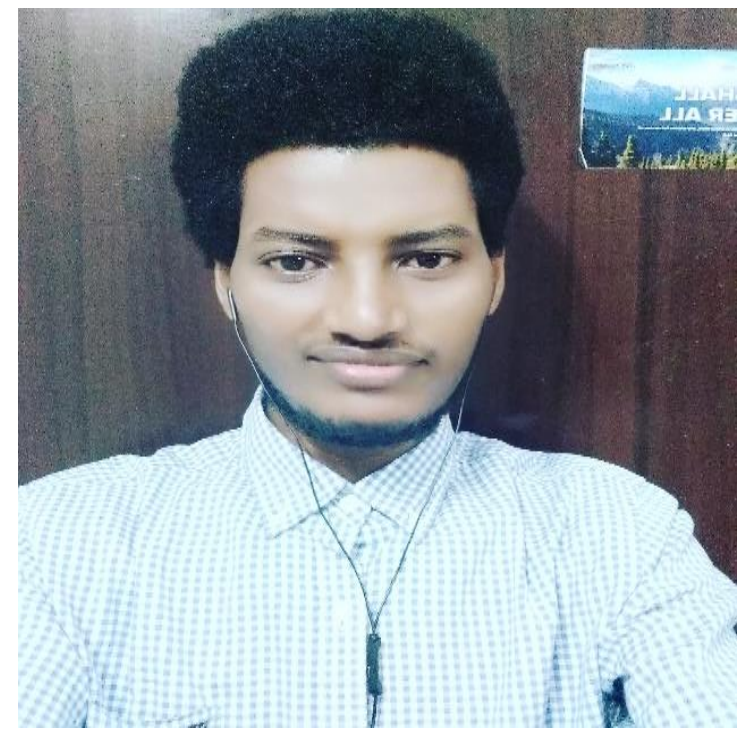

Milkessa Negeri Gemechu did his B.SC in Electronics and Communication Engineering at Arbaminch University Institute of Technology, Ethiopia. He has taught at Jigjiga University, Ethiopia as Assistant Lecturer, Worked in Ethiotelecom, Addis Abeba, Ethiopia and also taught at Mettu University, Oromiyaa, Ethiopia, as Assistant Lecturer, in addition to Serving as the Head of the Electrical and Computer Engineering Department. He did his M. Tech in Electronics and Communications Engineering (; Specializing in Systems and Signal Processing) at JNTU Hyderabad, Telangana, India. During his stay in India He has visited many Technology companies and got certificates of appreciation. He also actively worked with different charity and spiritual organizations getting valuable experiences and an Honorary awards for contributions.

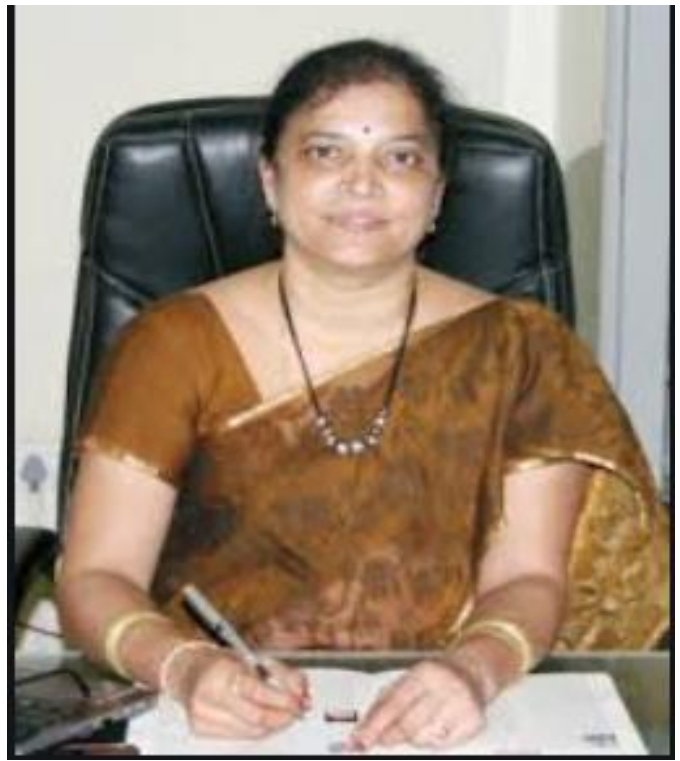

Dr. M. Madhavi Latha, Professor in the Department of ECE, Serving now as the University's Director of Academic and Planning. She has served at many key University leadership positions in her decades of teaching and research work. She has guided more than $100 \mathrm{~B}$. Tech projects, $210 \mathrm{M}$. Tech projects and 20 PHD Scholars, Published 178 papers in International/National conferences/journals. She is the recipient of "State Best Teacher Award" from Government of India, and also 'Exchange Visitor Award' from IEE-Department of States, USA, 'Best paper Award' and 'WIE Appreciation Award' from IEEE. She is also the Author a book, also having experience in Industry. She has also two patents officially registered with her own name.

CV:

https://jntuhceh.ac.in/faculty_portal/uploads/resumes/Madhavi\% 20Latha_Makkena_Profile.PDF 
